\title{
Cytokines and tryptophan metabolites can predict depressive symptoms in pregnancy
}

\author{
Qiong Sha ${ }^{1}$, Zach Madaj ${ }^{2}$, Sarah Keaton ${ }^{1,3}$, Martha L Escobar Galvis (iD ${ }^{1}$, LeAnn Smart (iD ${ }^{4}$, Stanislaw Krzyzanowski ${ }^{1}$, \\ Asgerally T. Fazleabas ${ }^{5}$, Richard Leach ${ }^{5}$, Teodor T. Postolache ${ }^{6,7,8,9}$, Eric D. Achtyes (D) ${ }^{3,4,10}$ and Lena Brundin (iD) $1,3,10^{凶}$
}

(c) The Author(s) 2022

Depression during and after pregnancy affects up to $20 \%$ of pregnant women, but the biological underpinnings remain incompletely understood. As pregnancy progresses, the immune system changes to facilitate fetal development, leading to distinct fluctuations in the production of pro-inflammatory factors and neuroactive tryptophan metabolites throughout the peripartum period. Therefore, it is possible that depression in pregnancy could constitute a specific type of inflammation-induced depression. Both inflammatory factors and kynurenine metabolites impact neuroinflammation and glutamatergic neurotransmission and can therefore affect mood and behavior. To determine whether cytokines and kynurenine metabolites can predict the development of depression in pregnancy, we analyzed blood samples and clinical symptoms in 114 women during each trimester and the postpartum. We analyzed plasma IL-1 $\beta, I L-2,-6,-8,-10, T N F$, kynurenine, tryptophan, serotonin, kynurenic- quinolinic- and picolinic acids and used mixed-effects models to assess the association between biomarkers and depression severity. IL-1 $\beta$ and IL- 6 levels associated positively with severity of depressive symptoms across pregnancy and the postpartum, and that the odds of experiencing significant depressive symptoms increased by $>30 \%$ per median absolute deviation for both IL- $1 \beta$ and IL- 6 (both $P=$ 0.01). A combination of cytokines and kynurenine metabolites in the 2 nd trimester had a $>99 \%$ probability of accurately predicting 3rd trimester depression, with an ROC AUC $>0.8$. Altogether, our work shows that cytokines and tryptophan metabolites can predict depression during pregnancy and could be useful as clinical markers of risk. Moreover, inflammation and kynurenine pathway enzymes should be considered possible therapeutic targets in peripartum depression.

Translational Psychiatry (2022)12:35; https://doi.org/10.1038/s41398-022-01801-8

\section{INTRODUCTION}

Peripartum depression (PPD) is defined as depression with onset during and up to four weeks after delivery [1]. In addition to symptoms of depression, which can range from mild to severely incapacitating, anxiety and suicidality are also frequently reported [2]. PPD in the mother is linked to low birth weight and prematurity [3], as well as emotional problems in the offspring, spanning from early childhood to adolescence [4]. Selective serotonin reuptake inhibitors (SSRIs) are the most common treatment for pregnancyrelated depression today, but they have been shown only to be effective in approximately 50\% of patients [5]. In addition, SSRIs are associated with certain risks during pregnancy, such as discontinuation syndrome in the newborn [6] and postpartum hemorrhage in the mother [7]. Physicians and mothers may therefore be reluctant to the use SSRIs, leaving this condition un- or undertreated.

The biological mechanisms underlying depressive symptoms during pregnancy remain to be fully elucidated. Several contributing factors have been suggested, including dysregulation of hormones, immune response, and aberrant genetic, or epigenetic factors [8]. Additionally, an increasing body of evidence suggests a role for inflammation in depression in general, and possibly specifically in PPD [9]. Due to the profound changes that the immune system undergoes during pregnancy it has been suggested that inflammation could play a particularly important role in the development of depressive symptoms during this period $[2,10]$. Several recent studies have shown an association between levels of pro-inflammatory cytokines in the blood of pregnant women and depressive symptoms [11-13].

The kynurenine pathway is the major enzymatic pathway that catabolizes tryptophan and has considerable immunomodulatory activity (Fig. 1). Interestingly, kynurenine pathway enzymes are highly expressed in the placenta, where their activity is key in the regulation of the maternal-fetal immune response [14]. The expression of indolamine-2,3-dioxygenase (IDO), the enzyme that catalyzes the transformation of tryptophan to kynurenine, as well as other pathway enzymes, increases with placental development [15]. In addition, it is known that the activity of the pathway is increased by interferon- $a$ and IL-6 [16]. Changes in activity are

\footnotetext{
${ }^{1}$ Department of Neurodegenerative Science, Van Andel Institute, Grand Rapids, MI, USA. ${ }^{2}$ Bioinformatics \& Biostatistics Core, Van Andel Institute, Grand Rapids, MI, USA. ${ }^{3}$ Division of Psychiatry \& Behavioral Medicine, Michigan State University College of Human Medicine, Grand Rapids, MI, USA. ${ }^{4}$ Pine Rest Christian Mental Health Services, Grand Rapids, MI, USA. ${ }^{5}$ Department of Obstetrics, Gynecology and Reproductive Biology, College of Human Medicine, Michigan State University, MI, USA. ${ }^{6}$ Mood and Anxiety Program, Department of Psychiatry, University of Maryland School of Medicine, Baltimore, MD, USA. ${ }^{7}$ Rocky Mountain MIRECC for Suicide Prevention, Aurora, CO, USA. ${ }^{8}$ Military and Veteran Microbiome Consortium for Research and Education, Aurora, CO, USA. ${ }^{9}$ Capital MIRECC, VISN 5, Baltimore, MD, USA. ${ }^{10}$ These authors contributed equally: Eric D. Achtyes, Lena Brundin. ${ }^{凶}$ email: lena.brundin@vai.org
}

Received: 22 September 2021 Revised: 5 January 2022 Accepted: 12 January 2022

Published online: 26 January 2022 


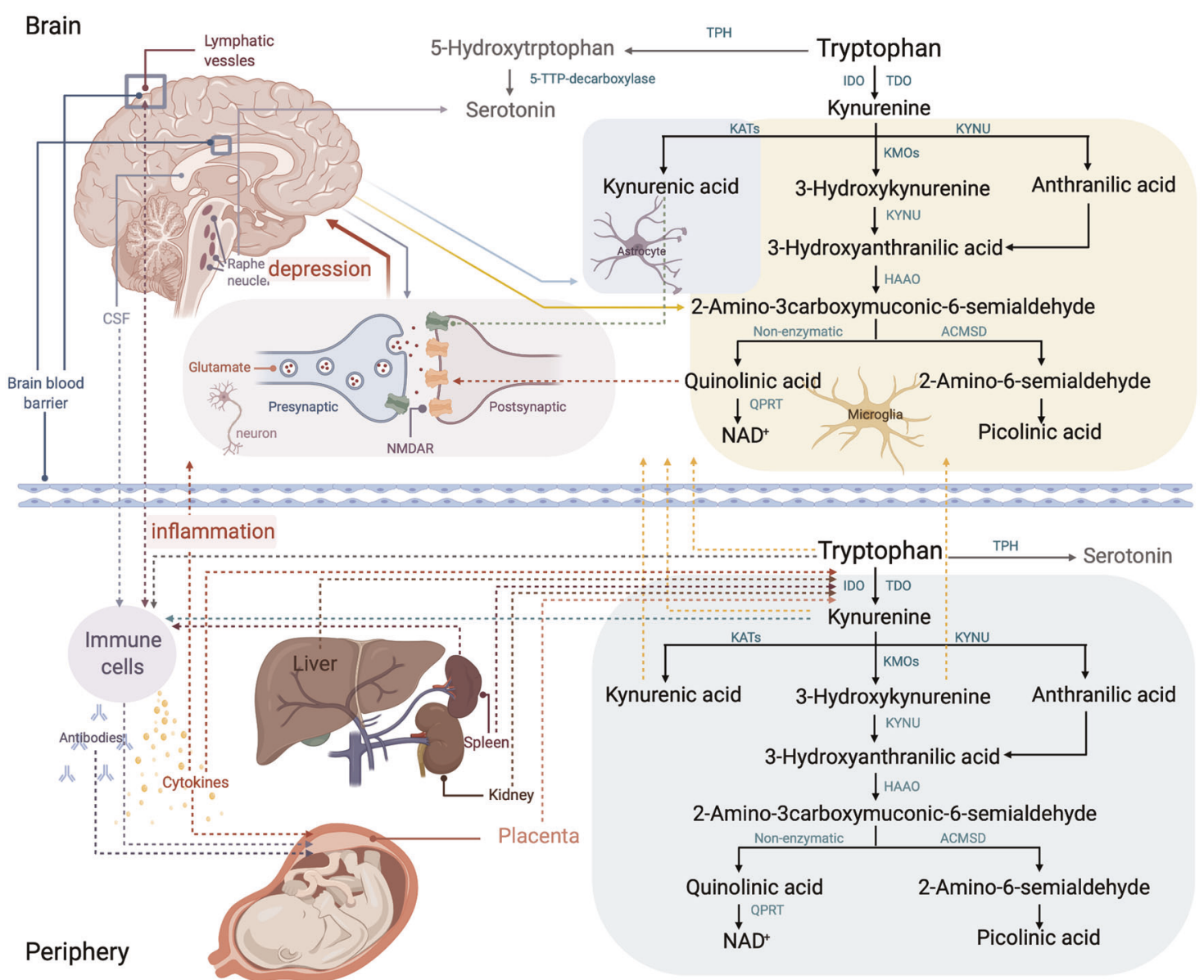

Fig. 1 Tryptophan metabolic pathways in the brain and periphery. Abbreviations: TPH tryptophan hydroxylase, IDO indoleamine 2,3dioxygenase. TDO tryptophan 2,3-dioxygenase. KATs kynurenine aminotransferases. KYNU kynureninase, HAAO 3-hydroxyanthranilate 3,4dioxygenase, ACMSD aminocarboxymuconate-semialdehyde decarboxylase, QPRT quinolinic acid phosphoribosyltransferase, CSF cerebrospinal fluid, NMDAR $N$-methyl-D-aspartate receptor, NAD nicotinamide.

important because several kynurenine pathway metabolites are neuroactive, including quinolinic acid (QUIN) and kynurenic acid (KYNA), both impacting glutamate neurotransmission [17]. Increased pathway activity could also affect serotonin levels, as tryptophan is also the substrate for its production (Fig. 1). Thus, it is plausible that changes in kynurenine pathway activity (and its metabolite levels) are causally linked to the generation of depressive symptoms as they can influence both central glutamatergic and serotonergic- mechanisms [18].

In spite of data pointing to an involvement of inflammatory mechanisms in pregnancy-related depression, longitudinal studies assessing how levels of inflammatory factors and kynurenine pathway metabolites fluctuate during pregnancy are sparse. Also, it is not known whether an increased production of these molecules can predict the development of depressive symptoms. Such information would be important for developing biomarkers to help clinicians identify women at risk, possibly even before symptoms manifest. We hypothesized that the levels of pro-inflammatory cytokines and neuroactive kynurenine metabolites would be positively linked to the severity of depressive symptoms, and that single markers, or a combination thereof, measured early in pregnancy could serve as biomarkers to predict subsequent depression.

\section{MATERIALS AND METHODS}

\section{Study design}

This study was approved by the Institutional Review Board (IRB) of Michigan State University, East Lansing, with reciprocal approvals by Van
Andel Research Institute IRB and Spectrum Health IRB, both in Grand Rapids, Michigan. Informed consent was obtained from all subjects. Pregnant women were enrolled in their first trimester, returning each trimester and in the postpartum for psychiatric assessments and blood sampling. Inclusion criteria for enrollment were: all races and national origins of pregnant females, age 18 and above, English speaking and able to give informed consent as well as able to comply with and complete study procedures. Our exclusion criteria were: nonpregnant females, patients with psychotic symptoms and/or severe cognitive impairment that interfere with their ability to give informed consent or to complete study assessments. Patients that could not read and write in English as research measures used have only been validated in English-speaking populations. Patients that had blood-borne chronic infections including hepatitis B, C, or HIV as established at routine pregnancy blood screens. Patients who had any schizophrenia spectrum disorder or bipolar disorder type 1 (based on self-report and SCID interview). Patients who reported ongoing substance abuse or dependence (in the past 3 months). Three patients had a systemic autoimmune disease (systemic lupus erythematosus and myasthenia gravis) and were subsequently excluded, leaving 114 women in the final cohort.

\section{Clinical cohort}

The women were recruited from the Obstetrics and Gynecology clinic at Spectrum Health, Grand Rapids, Michigan, between 2015-2018. The mean age of the women was $25( \pm 5.9)$ at the beginning of the study. All the participants had high school level education or above, with $16 \%$ obtaining a bachelor's degree. More than $60 \%$ of the women lived in a household with an annual income of less than $\$ 34,000$. Our cohort was ethnically diverse, with over $50 \%$ non-White/Caucasian. Caucasian was the largest individual group at $47 \%$, followed by Black/African American at $26 \%$. We 
Table 1. Patient demographics.

\begin{tabular}{|c|c|c|}
\hline & $\begin{array}{l}\text { Median/ } \\
\text { Frequency }\end{array}$ & SD \% \\
\hline Age & 25 & \pm 5.9 \\
\hline BMI & 29 & \pm 8.9 \\
\hline Taking an SSRI (\%) & 4 & $3.5 \%$ \\
\hline Employed \% & 64 & $56.1 \%$ \\
\hline \multicolumn{3}{|l|}{ Race } \\
\hline American Indian or Alaskan Indian & 2 & $1.8 \%$ \\
\hline White/Caucasian & 54 & $47.4 \%$ \\
\hline Black or African American & 30 & $26.3 \%$ \\
\hline Multiracial & 12 & $10.5 \%$ \\
\hline Other & 16 & $14 \%$ \\
\hline \multicolumn{3}{|l|}{ Highest education } \\
\hline Some high school & 19 & $16.7 \%$ \\
\hline High school diploma & 31 & $27.2 \%$ \\
\hline $\begin{array}{l}\text { Technical/trade/ allied health training } \\
\text { degree or certification }\end{array}$ & 5 & $4.4 \%$ \\
\hline Some college & 41 & $36 \%$ \\
\hline Bachelor's degree or higher & 18 & $15.8 \%$ \\
\hline \multicolumn{3}{|l|}{ Annual household income } \\
\hline$\$ 15,000$ or less & 41 & $36 \%$ \\
\hline$\$ 15,001-\$ 34,000$ & 31 & $27.2 \%$ \\
\hline$\$ 34,001-\$ 70,000$ & 21 & $18.4 \%$ \\
\hline$\$ 70,001-\$ 120,000$ & 10 & $8.8 \%$ \\
\hline$\$ 120,000$ or more & 8 & $7.0 \%$ \\
\hline \multicolumn{3}{|l|}{ Marital status } \\
\hline Married or living with someone & 46 & $40.4 \%$ \\
\hline Single, unmarried & 59 & $51.8 \%$ \\
\hline Have a partner, not living together & 8 & $7.0 \%$ \\
\hline
\end{tabular}

also had study participants of American Indian, Alaskan Indian and multiracial ethnicity (Table 1). The majority of the women in the study were single or unmarried; $40 \%$ were married or living with someone (Table 1); $42 \%$ of the women had no previous children in the household, $30 \%$ had one child and the rest of the participants had two to five children.

\section{Patient evaluation}

The Edinburgh Postnatal Depression Rating Scale (EPDS) was used to measure depressive symptom severity [19] at each of the four visits. The Structured Clinical Interview for DSM-IV (SCID) was administered by a trained clinician at baseline, and then again whenever a subject scored $>10$ on the EPDS, to establish (or rule out) a new diagnosis of major depressive disorder with peripartum onset (PPD), as defined by DSM-5 [20]. An EPDS score $\geq 13$ over the last 7 days has been shown to be a valid indicator of depression during pregnancy and the first postpartum year [21] and was thus used as dichotomous variable (EPDS $\geq 13$ and $<13$ ) in this study. A somatic health report, including comorbid medical conditions, medications, vital signs (BMI, pulse, blood pressure, temperature), and a blood draw were also completed at all visits.

\section{Plasma cytokine analysis}

Blood samples were collected by venous puncture into EDTA tubes, between 9:00 am and 12:00 pm and immediately transported to the laboratory on ice. Samples were centrifuged into plasma $\left(700 \times g\right.$ at $\left.4{ }^{\circ} \mathrm{C}\right)$, aliquoted and immediately frozen at $-80^{\circ} \mathrm{C}$ until analysis. IL-1 $\beta, \mathrm{IL}-2, \mathrm{IL}-6$, IL-8, IL-10, and TNF levels were run on a Sector 600 (K15049D, Meso Scale Diagnostics, Rockville, MD, USA) following manufacturer's instructions as previously published [2]. Samples were run in duplicate and mean values were used for analysis. Inter-assay coefficients of variation (\% CV) were: IL$1 \beta$ (3.5\%), IL-2 (3.0\%), IL-6 (2.2\%), IL-8 (2.4\%), IL-10 (1.8\%), and TNF (2.8\%).
Detection of tryptophan, serotonin, and kynurenine pathway metabolites

The laboratory analyses were performed by staff that were blinded for all clinical parameters. A research number was assigned to each sample to enable future integration with clinical data. Tryptophan (TRP), serotonin (SERO), kynurenine (KYN), KYNA, QUIN and picolinic acid (PIC) were analyzed as previously described [2]. Briefly, plasma TRP, SERO and KYN concentrations were measured using high-performance-liquid chromatography with ultraviolet/visible wavelength detection and results were analyzed by Chromeleon 7.2 Chromatography Data System (Thermo Scientific, Dionex). QUIN and PIC were separated by gas chromatographymass spectrometry (GC-MS) and quantified using Thermo Xcaliber and Waters Masslynx software. Ultra-high-performance-liquid-chromatography, coupled to mass spectrometry (UPLC-MS/MS) was used to measure KYNA concentrations. Analytical conditions were set according to a previously published protocol [22]. Three derivative predictors-metabolite ratioswere created by division, KYN/TRP (rKT), QUIN/KYNA (rQK) and QUIN/PIC (rQP). These ratios are used to express the differential activity of specific pathway enzymatic steps/branches. KYN/TRP is a proxy of the activity of the first step of the pathway, QUIN/KYNA is often called the "neurotoxic ratio" and can be viewed as a proxy for the glutamate agonism of QUIN vs the antagonist KYNA, and QUIN/PIC is a proxy for ACMSD activity, the enzyme that produces PIC at the expense of QUIN (Fig. 1).

\section{Statistical analysis}

Statistical analyses were conducted using RStudio 4.0.2 (https://cran.rproject.org/). The sample size was calculated to ensure adequate power to detect a prespecified effect size of $\geq 0.8$ based on kynurenine metabolite measurements in plasma from psychiatric patients [23]. Concentrations of biomarkers were natural log-transformed when applicable to meet model assumptions. The following number of individuals without missing data for at least one of the analyses were included at each timepoint: 112 (1st trimester), 88 (2nd trimester), 82 (3rd trimester), 78 (postpartum). 56 women had data from all timepoints.

Associations between depressive symptoms and biomarkers across time were evaluated by ordinal mixed-effects regression, and logistic mixedeffects regression was used to evaluate the risk of experiencing significant depressive symptoms (binary outcome: EPDS $\geq 13$ ). Bayesian generalized (non)-linear ordinal multilevel models and Bayesian logistic regression [24], both adjusted for age, were used to predict subsequent EPDS scores (ordinal) and EPDS $\geq 13$ (binary) respectively. For both ordinal and logistic analyses, a full model comprised of the principal components that explained $\geq 10 \%$ of the markers' variance and a horseshoe prior was initially fit to maximize predictive capacity while avoiding multicollinearity and overfitting. Model performance was evaluated via leave-one-out (LOO) cross-validation by comparing the full model with age-only and null, intercept-only models [25]. Bayesian ordinal and logistic models with flat priors were also built for each of the top markers in the first principal component to compare with the full model. Predictive accuracy was visualized by ROC and PR curves [26]. Marginal effect plots from individual biomarkers by age were generated based on the Bayesian models to visualize predicted PPD diagnoses.

\section{RESULTS}

\section{Characteristics of the cohort}

The demographic characteristics of the participants are summarized in Table 1. The average age was 26 years (18-44 range). 37 out of the 114 women (32\%) experienced significant depressive symptoms (EPDS $\geq 13$ ) at one or more timepoints and 12 women (10.5\%) received a formal diagnosis of PPD via DSM SCID interview during the study. The percentage of women experiencing significant depressive symptoms (EPDS score of $\geq 13$ ) was $19.3 \%, 13.6 \%, 15.8 \%$, and $14.1 \%$ during the $1 \mathrm{st}, 2 \mathrm{nd}$, and $3 \mathrm{rd}$ trimester, and in the postpartum, respectively. Depressive symptoms and the median levels of the biomarkers at each timepoint are shown in Table. S1.

\section{Biomarkers significantly correlated with depression severity and risk}

IL1 $\beta$, IL-6 and QUIN, were significantly associated with depression severity and/or higher odds of having an EPDS $\geq 13$. Higher levels 
Table 2. Biomarker contribution to -and correlation with- PC1 during the 2nd trimester.

\begin{tabular}{|c|c|c|c|c|c|c|c|}
\hline \multirow[t]{2}{*}{ Biomarker } & \multirow{2}{*}{$\begin{array}{l}\text { Contribution } \\
\text { to PC1 (\%) }\end{array}$} & \multirow{2}{*}{$\begin{array}{l}\text { Weight to } \\
\text { PC1 (MAD) }\end{array}$} & \multirow{2}{*}{$\begin{array}{l}\text { PC1 Spearman Rho } \\
\text { (FDR adjusted } p \text { value) }\end{array}$} & \multicolumn{4}{|c|}{ 3rd trimester acute EPDS: Percent change in OR ( $95 \%$ credible interval) } \\
\hline & & & & EPDS & $\begin{array}{l}\text { Posterior } \\
\text { probability }\end{array}$ & EPDS $\geq 13$ & $\begin{array}{l}\text { Posterior } \\
\text { probability }\end{array}$ \\
\hline TNF & 18.67 & 0.54 & $0.789(<0.0001)$ & $53.7(18.5,105.4)$ & $>0.99$ & $33.6(0,94.6)$ & 0.95 \\
\hline $\mathrm{rKT}^{\mathrm{a}}$ & 18.12 & 0.53 & $0.768(<0.0001)$ & $51.7(22.1,87.8)$ & $>0.99$ & $52.2(12.7,116.0)$ & 0.99 \\
\hline QUIN & 15.17 & 0.48 & $0.713(<0.0001)$ & $78.6(28.4,150.9)$ & $>0.99$ & $91.6(15.0,232.0)$ & 0.98 \\
\hline KYN & 14.23 & 0.47 & $0.572(<0.0001)$ & $56.8(23.4,99.4)$ & $>0.99$ & $52.2(8.3,120.3)$ & 0.98 \\
\hline IL-10 & 5.9 & 0.3 & $0.330(0.0022)$ & $5.1(-5.8,18.5)$ & 0.76 & $5.1(-12.2,25.9)$ & 0.72 \\
\hline IL-6 & 5.52 & 0.29 & $0.465(<0.0001)$ & $64.9(32.3,107.5)$ & $>0.99$ & $58.4(22.1,111.7)$ & $>0.99$ \\
\hline rQP & 5.3 & 0.29 & $0.639(<0.0001)$ & $19.7(-16.5,73.3)$ & 0.79 & $19.7(-22.1,80.4)$ & 0.78 \\
\hline
\end{tabular}

Correlation between biomarkers in the 2nd trimester and EPDS scores from the 3rd trimester via Bayesian's prediction models. Biomarkers were standardized via Robust Standardization. One PC1 unit corresponds to a 0.54 MAD (median absolute deviation) change in TNF, a 0.53 MAD change in rKT, a 0.48 MAD change in QUIN, a 0.47 MAD change in KYN, a 0.36 MAD change in $\mathrm{RQK}$, a $0.3 \mathrm{MAD}$ change in IL-10, a 0.29 MAD change in IL-6, and a $0.29 \mathrm{MAD}$ change in rQP. arKT: odds ratio from $1 / 100$ increase in rKT.

Bolded text indicates $>95 \%$ chance marker has a true association with EPDS or EPDS $>=13$.

of IL-6 were found to be associated with increased depression severity and risk of experiencing significant depressive symptoms at all timepoints $(P=0.01$ Table. S2). Increasing levels of IL-1 $\beta$ were further associated with worsening depressive symptoms (EPDS, ordinal regression $P=0.02$ ) and an elevated risk of experiencing significant depressive symptoms (EPDS $\geq 13$, logistic regression $P=0.01)$ with an OR increase of $32.3 \%(95 \% \mathrm{Cl}$ : 7.0 , 63.6) per median absolute deviation (MAD) of IL-1 $\beta$ (Table. S2). The association between QUIN and depression was also robust, as higher levels in the 3rd trimester were associated with both increasing depression severity ( $P=0.04$, EPDS score) and risk of falling into the category of significant symptoms (EPDS $\geq 13$ ) with an OR increase $41.5 \%$ per MAD; $95 \% \mathrm{Cl}$ : 1.8, 96.6; $P=0.02$ (Table. S2).

\section{Principle component analysis (PCA)}

We assessed the capacity of the biomarkers at one timepoint to predict depressive symptoms at the following timepoint Table 2. To prevent multicollinearity among all the biomarkers from influencing the model, a PCA was conducted to reduce the number of predictors into a smaller set of independent covariates. All principal components (PCs) that explained $>10 \%$ of the total variance among all markers (6 cytokines, 4 kynurenine metabolites, tryptophan, serotonin and 3 derivative ratios) were included in the final model (PC1 to PC5). The top contributors to PC1 were TNF, rKT, QUIN, KYN, rQK, IL-10, IL-6, rQP; they cumulatively explain $>90 \%$ of the variance of PC1 (Table 2). These markers were included in the subsequent analyses.

\section{Prediction of depressive symptoms utilizing individual markers}

IL-6, rKT, QUIN, and KYN measured in the 2nd trimester have strong evidence (>95\% chance) of being positively associated with both depression severity (EPDS) and risk of significant depressive symptoms (EPDS $\geq 13$ ) in the 3rd trimester. Also, TNF and rQK in the 2nd trimester, were strongly associated with depression severity in the 3rd trimester (EPDS) (Table 2).

In the ROC analysis, IL- 6 had the best performance among the individual biomarkers used to predict depressive symptoms (AUC $=0.79$ and 0.8 by Bayesian ordinal and logistic regression, respectively). Kynurenine, QUIN and rKT also produced good predictions with six out of seven markers having a ROC AUC $>0.7$ by either model (Fig. S1A, C). Precision recall analyses were also estimated and confirmed the predictive value of our model (Fig. S1B, D).

\section{Prediction of depression severity at future timepoints using multiple markers}

To estimate the performance of our predictive algorithm on data not used to train our model, we first performed a leave-one-out cross validation, which indicated that our predictability, as could be generalized to other cohorts from this data set, would be optimal from mid- to late pregnancy (2nd to 3rd trimester, see Table. S3).

The prediction from 2nd to 3rd trimester is illustrated in Fig. 2 (ROC- and PR curves). For both models (predicting EPDS and EPDS $\geq 13$ ), the first principal component (PC1) had $>99 \%$ chance of being positively associated with depression severity and $>94 \%$ for the depressive phenotype (Bayes Factors $=265$ and 17, respectively). For each unit increase in $P C 1$, the odds of experiencing significant depressive symptoms increased by $31 \%$ (95\% Cl: 0, 77; Table 2). Based on the area under ROC- and PR curves, the full model nominally outperformed each individual marker for predicting risk of significant depressive symptoms. Both ordinal and logistic regression full models had ROC AUC = 0.83, PR AUC $=0.41$ (Fig. 2).

\section{PPD diagnosis and usefulness of biological "depression- warning" triggers}

As a proof-of-concept pilot, we established whether 12 patients diagnosed with PPD using SCID interviews during pregnancy would have been identified using our proposed biomarker panel, derived from the 2nd trimester, where we found most evidence for generalizability. To this end, we generated an age-adjusted "depression-warning" level for the four main risk biomarkers identified in our statistical models (IL-6, TNF, kynurenine and QUIN). The biomarker level chosen to trigger "depressionwarning" was set as the level where the probable EPDS score was $>10$ at each age of the women, the same level that triggered a SCID diagnostic interview in this study. The depression-warning levels per age for IL-6, TNF, QUIN and kynurenine are visualized in Fig. 3. Using these biological "depression-warning" measurements, we would have identified $83.3 \%$ of all SCID diagnosed cases, based on only the biomarker measures. Adding the rKT ratio to the biomarkers did not further increase the test's sensitivity.

\section{DISCUSSION}

We established that inflammatory cytokines, in particular IL-6 and $\mathrm{IL}-1 \beta$, as well as kynurenine pathway metabolites, are associated with the severity of depressive symptoms in pregnancy and the 

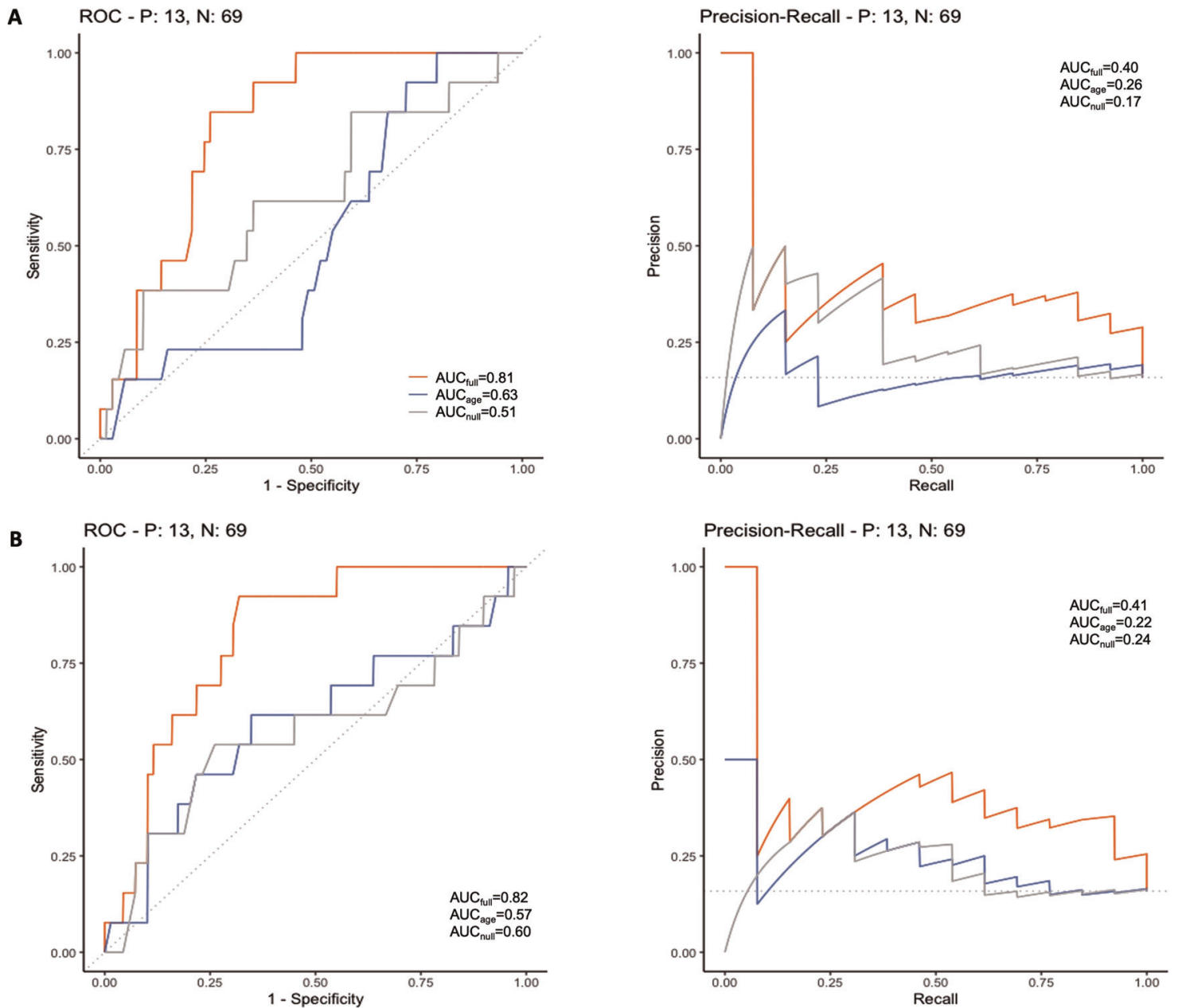

Fig. 2 ROC and PR curves of the prediction model comparisons. ROC and PR curves of the prediction model comparisons (between full, ageonly and null models) using biomarkers collected during the 2 nd trimester to predict 3rd trimester EPDS $\geq 13$. A using ordinal regression (cumulative probability that EPDS $\geq 13$ ), B logistic regression (estimated probability EPDS $\geq 13$ ). The full model outperforms the other two models in both the ROC and PR curves. The performance of the PR curve is important when the number of cases (EPDS $\geq 13$ ) is not equal to non-cases (EPDS < 13). The leave-one-out cross-validation results are also consistent with the full model having the best predictive accuracy.

postpartum, and that these markers were useful to predict the future development of depression during pregnancy. Our full model, composed of all 15 biomarkers (including three ratios), had an AUC of more than $83 \%$ for predicting depression as defined by EPDS $\geq 13$. Moreover, IL-6, QUIN, TNF, and KYN showed strong individual accuracy in predicting depression risk and severity at future timepoints, with the best performance using the 2nd trimester markers to predict symptoms emerging in the $3 \mathrm{rd}$ trimester. In our proof-of-concept pilot, we found that we would have identified $83 \%$ of PDD-diagnosed women (identified by SCID interviews) by means of the biomarkers only. Certainly, further evaluation in larger cohorts will be necessary to refine and fully estimate the specificity, sensitivity, and positive predictive value of these biomarkers. Additionally, the analytical methods and reagents need to be cross-validated and standardized across multiple sites to allow for any broad implementation.

During pregnancy and postpartum women experience major immune function fluctuations. These immunological changes are part of the women's adaptation to the fetal development and are critical for both maternal and infant health. During a normal pregnancy, the maternal immune system goes through several physiological stages, including an early, more pro-inflammatory state, important for implantation and placentation, characterized by increased production of cytokines, chemokines and growth factors; next, comes an anti-inflammatory phase, characterized by rapid fetal growth; and lastly, another pro-inflammatory phase, preceding delivery in preparation for parturition [27]. During the postpartum phase, the body gradually returns to a nonpregnant homeostasis, as it relates to immune regulation [28]. The physiological fluctuations in inflammatory factors can impact emotion and behavior, and may do so in pregnancy [29]. However, we propose that when these changes become extreme, or take place in women vulnerable to inflammation-induced depression, the mood changes can contribute to the pathophysiological processes leading to PPD. It is interesting to note that the markers we examined here showed predictive capacity during pregnancy, but not for the postpartum. This likely indicates that partially differential mechanisms, such as hormonal changes linked to parturition and possibly lactation, also influence the development of depression during this period [30].

In addition to their role as biomarkers of disease, the analytes measured in this study have important biological functions that can be part of the molecular underpinnings of depressive symptoms. The metabolites of the kynurenine pathway have both immunoregulatory, inflammatory and neuroactive properties [18]. QUIN, in particular, was a significant predictor of future depressive symptoms from mid- to late pregnancy. This metabolite is an endogenous modulator of glutamate receptors, with agonist properties on the $\mathrm{N}$-methyl-D-aspartate receptor (NMDAR) [17]. QUIN also has additional effects, including increasing 

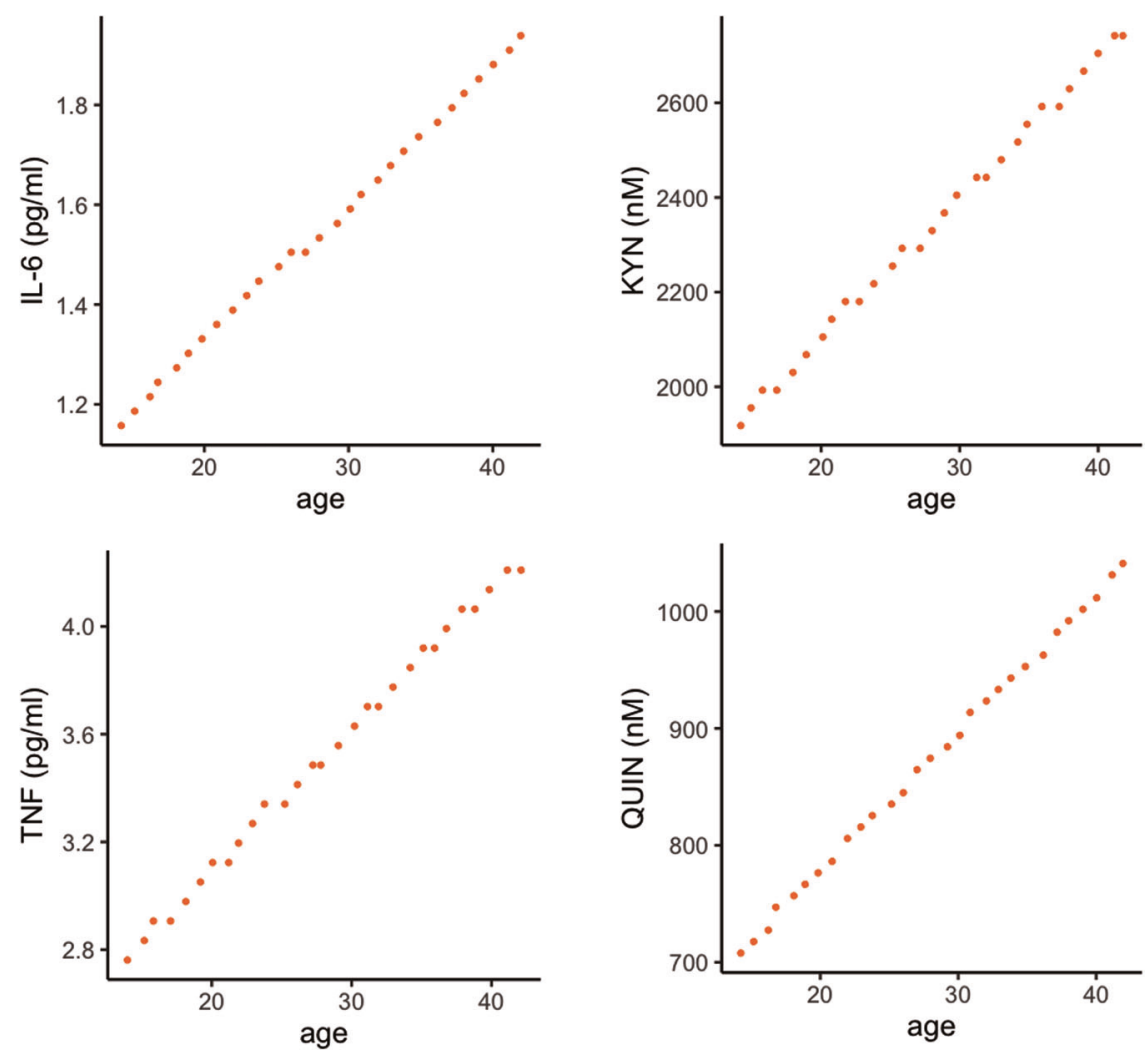

Fig. 3 Depression-warning cut off per age. Bayesian models with flat priors were used to predict the probability of each 3rd trimester EPDS score based on individual marker levels and age. Plotted here are the minimal marker levels where the most probable EPDS score is $\geq 10$ for women ages 18-44. As marker levels exceed these plotted thresholds, the probability that a given woman would score $\geq 10$ on the EPDS scale in the $3^{\text {rd }}$ trimester continues to increase asymptotically to 1 . An EPDS score of 10 or greater is used clinically to screen women for PPD, thus these plots present a proof-of-concept for an advanced screening method where a woman's age and measures of these 4 markers could jointly be used to flag someone as likely to score $\geq 10$ on the EPDS assessment in the next trimester and therefore considered to be at risk of developing future PPD.

glutamate release while inhibiting its reuptake by astrocytes, both of which have stimulatory effects on glutamatergic neurotransmission [31]. Peripheral levels of cytokines and kynurenine metabolites are known to reflect the central levels, as our group and others have shown significant correlations between the blood and CSF levels of acute-phase reactants, inflammatory cytokines and kynurenine metabolites, including QUIN $[32,33]$. Although in this study, we did not draw any CSF samples from the pregnant women, several studies have shown evidence that peripheral inflammation and kynurenine pathway activation are closely mirrored in the central compartment $[32,34,35]$. Therefore, it is plausible that the increased inflammation and pathway activation we observe in the periphery, could reflect central mechanisms underlying symptom generation.

Previous studies in depression and suicidality have found increased levels of cytokines, in particular IL-6, and kynurenine metabolites, in blood and CSF of patients with depression and suicidality [36]. Additionally, experimental studies confirmed that alterations in inflammation and kynurenine metabolism can lead to depressive-like behavior. This has been established in animal models [37] and human volunteers injected with endotoxin to stimulate inflammation [38]. It is known that interferon- $a$ injections, to treat hepatitis $C$, lead to depressive symptoms, together with an increase in cytokines and QUIN in the CSF [35]. Interestingly, in an experimental study using mice, depressive-like effects of inflammation were fully reversed by modulating the NMDAR following ketamine treatment [39]. In the current study, the finding that these biomarkers not only correlate with depressive symptoms, but also might predict future symptom development, further strengthens the causal inference that these metabolites and cytokines might contribute to the development of depressive symptoms, in addition to being biomarkers of disease. Consequently, these mechanisms could be considered in the future therapeutic development for PPD. As such, it will also be important to understand the factors contributing to the inflammation and kynurenine pathway activation that we observe in women with PPD. Examples of contributing mechanisms could include lack of vitamin D, infections, changes in gonadal hormones or cortisol [40], as well as dietary factors [41]. Modulating the upstream causes of inflammation may be feasible in certain women, while treating the downstream pathways triggered by inflammation might be optimal in others.

To our knowledge, this is the first longitudinal study examining the ability of a panel of mechanistically implicated cytokines and tryptophan metabolites to predict the development of depression in pregnancy. Predicting which individuals may be at risk for depression during pregnancy is a difficult task for clinicians. The importance of detecting depression in pregnant women has been highlighted by American College of Obstetricians and Gynecologists [42], which encourages self-assessments using the EPDS when women are in contact with health care professionals during and after pregnancy. Reporting of depressive symptoms in this period may also be linked with feelings of shame or guilt due to cultural stigma, leading to an underestimation of symptom prevalence and severity and reduced help-seeking [43]. Importantly, the addition of biological markers as tools to predict future depression risk, acting as "depression-warning" signs, could help clinicians schedule more frequent follow-up visits, consider stressreduction and psychotherapy, include supportive or cognitivebehavioral techniques, or possibly intervene with preventative 
measures (e.g., use of anti-inflammatories) before worrisome symptoms begin.

Limitations of this study include that it was conducted at a single center, which could reduce generalizability. It is however important to note that our cohort was relatively diverse, $<50 \%$ White/Caucasian (Table 1). Also, the sample size was moderate for a study of this type (>350 samples were analyzed, over four timepoints). Therefore, these findings warrant further replication in larger cohorts to establish whether the biomarkers can be developed into a clinically useful predictive panel for depression risk in pregnant women. Additional biomarkers, possibly including neopterin, cortisol and additional cytokines, could also be assayed to determine whether the accuracy of prediction could be improved even further. Despite the limitations, our current study provides important insights into the possible role of inflammatory biomarkers in perinatal depression.

In conclusion, we established that inflammatory cytokines and kynurenine metabolites are associated with depression severity during pregnancy and the postpartum. Together with the data from both clinical and animal models demonstrating a causal role for these biological mediators, inducing depression and depressive-like behavior [44, 45], our longitudinal data suggest that our analytes could also be causal mediators in pregnancyrelated depression. Randomized controlled experiments are necessary to confirm this hypothesis, as an initial step in assessing the possibility of treating inflammation in PPD; caution must however be taken due to the involvement of these factors in fetal development. Importantly, our study showed that a set of biomarkers was able to predict the future development of depression during pregnancy, and steps could be taken to move these markers into clinical risk assessments.

\section{REFERENCES}

1. Gavin NI, Gaynes BN, Lohr KN, Meltzer-Brody S, Gartlehner G, Swinson T. Perinatal depression: a systematic review of prevalence and incidence. Obstet Gynecol. 2005;106:1071-83.

2. Achtyes E, Keaton SA, Smart L, Burmeister AR, Heilman PL, Krzyzanowski S, et al. Inflammation and kynurenine pathway dysregulation in post-partum women with severe and suicidal depression. Brain, Behav, Immun. 2019;83:139-247.

3. Becker M, Weinberger T, Chandy A, Schmukler S. Depression during pregnancy and postpartum. Curr Psychiatry Rep. 2016;18:32.

4. Rogers A, Obst S, Teague SJ, Rossen L, Spry EA, Macdonald JA, et al. Association between maternal perinatal depression and anxiety and child and adolescent development: a meta-analysis. JAMA Pediatrics. 2020;174:1082-92.

5. De Crescenzo F, Perelli F, Armando M, Vicari S. Selective serotonin reuptake inhibitors (SSRIs) for post-partum depression (PPD): a systematic review of randomized clinical trials. J Affect Disord. 2014;152-154:39-44.

6. Levinson-Castiel R, Merlob P, Linder N, Sirota L, Klinger G. Neonatal abstinence syndrome after in utero exposure to selective serotonin reuptake inhibitors in term infants. Arch Pediatrics Adolesc Med. 2006;160:173-6.

7. Hanley GE, Smolina K, Mintzes B, Oberlander TF, Morgan SG. Postpartum hemorrhage and use of serotonin reuptake inhibitor antidepressants in pregnancy. Obstet Gynecol. 2016;127:553-61.

8. Yim IS, Tanner Stapleton LR, Guardino CM, Hahn-Holbrook J, Dunkel, Schetter C. Biological and psychosocial predictors of postpartum depression: systematic review and call for integration. Annu Rev Clin Psychol. 2015;11:99-137.

9. Lee $\mathrm{C}-\mathrm{H}$, Giuliani $\mathrm{F}$. The role of inflammation in depression and fatigue. Front Immunol. 2019;10:1696.

10. Christian LM, Franco A, Glaser R, lams JD. Depressive symptoms are associated with elevated serum proinflammatory cytokines among pregnant women. Brain, Behav, Immun. 2009;23:750-4.

11. Mehta D, Grewen K, Pearson B, Wani S, Wallace L, Henders AK, et al. Genomewide gene expression changes in postpartum depression point towards an altered immune landscape. Transl Psychiatry. 2021;11:155.

12. Lahti-Pulkkinen $M$, Girchenko $P$, Robinson R, Lehto SM, Toffol E, Heinonen $K$, et al. Maternal depression and inflammation during pregnancy. Psychological Med. 2020;50:1839-51.

13. Petralia Cristina M, Mazzon E, Fagone $P$, Falzone $L$, Bramanti $P$, Nicoletti $F$, et al. Retrospective follow-up analysis of the transcriptomic patterns of cytokines, cytokine receptors and chemokines at preconception and during pregnancy, in women with post-partum depression. Exp Ther Med. 2019;18:2055-62.

14. Sedlmayr P, Blaschitz A, Stocker R. The role of placental tryptophan catabolism. Front Immunol. 2014;5:230.

15. Tatsumi K, Higuchi T, Fujiwara H, Nakayama T, Egawa $H$, Itoh K, et al. Induction of tryptophan 2,3-dioxygenase in the mouse endometrium during implantation. Biochemical biophysical Res Commun. 2000;274:166-70.

16. Dantzer R. Role of the kynurenine metabolism pathway in inflammation-induced depression: preclinical approaches. Curr Top Behav Neurosci. 2017;31:117-38.

17. Stone TW. Neuropharmacology of quinolinic and kynurenic acids. Pharmacol Rev. 1993:45:309-79.

18. Schwarcz R, Bruno JP, Muchowski PJ, Wu H-Q. Kynurenines in the mammalian brain: when physiology meets pathology. Nat Rev Neurosci. 2012;13:465-77.

19. Di Florio A, Putnam K, Altemus M, Apter G, Bergink V, Bilszta J, et al. The impact of education, country, race and ethnicity on the self-report of postpartum depression using the Edinburgh Postnatal Depression Scale. Psychological Med. 2017;47:787-99.

20. American Psychiatric Association. Diagnostic and statistical manual of mental disorders. In: Diagnostic and statistical manual of mental disorders. Arlington, VA, 2013.

21. Rubertsson C, Börjesson K, Berglund A, Josefsson A, Sydsjö G. The Swedish validation of Edinburgh Postnatal Depression Scale (EPDS) during pregnancy. Nord J Psychiatry. 2011;65:414-8.

22. Cellar NA, McClure SC, Salvati LM, Reddy TM. A new sample preparation and separation combination for precise, accurate, rapid, and simultaneous determination of vitamins B1, B2, B3, B5, B6, B7, and B9 in infant formula and related nutritionals by LC-MS/MS. Analytica Chim Acta. 2016;934:180-5.

23. Brundin L, Sellgren CM, Lim CK, Grit J, Pålsson E, Landén $M$, et al. An enzyme in the kynurenine pathway that governs vulnerability to suicidal behavior by regulating excitotoxicity and neuroinflammation. Transl Psychiatry. 2016;6: e865-e865.

24. Bürkner P-C brms: An R package for Bayesian multilevel models using Stan Journal of Statistical Software; 80:1.

25. Vehtari A, Gelman A, Gabry J. Practical Bayesian model evaluation using leaveone-out cross-validation and WAIC. Stat Comput. 2017;27:1413-32.

26. Sing T, Sander O, Beerenwinkel N, Lengauer T. ROCR: visualizing classifier performance in R. Bioinformatics. 2005;21:3940-1.

27. Mor G, Cardenas I, Abrahams V, Guller S. Inflammation and pregnancy: the role of the immune system at the implantation site. Ann N. Y Acad Sci. 2011;1221:80-87.

28. Groer ME, Jevitt $C$, Ji M. Immune changes and dysphoric moods across the postpartum. Am J Reprod Immunol. 2015;73:193-8.

29. Leff-Gelman P, Mancilla-Herrera I, Flores-Ramos M, Cruz-Fuentes C, Reyes-Grajeda JP, García-Cuétara MDP, et al. The immune system and the role of inflammation in perinatal depression. Neurosci Bull. 2016;32:398-420.

30. Payne JL, Maguire J. Pathophysiological mechanisms implicated in postpartum depression. Front Neuroendocrinol. 2019;52:165-80.

31. Lugo-Huitrón R, Ugalde Muñiz P, Pineda B, Pedraza-Chaverrí J, Ríos C, Pérez-de la Cruz V Quinolinic acid: an endogenous neurotoxin with multiple targets. Oxidative Medicine and Cellular Longevity 2013;104024.

32. Heilman PL, Wang EW, Lewis MM, Krzyzanowski S, Capan CD, Burmeister AR, et al. Tryptophan metabolites are associated with symptoms and nigral pathology in Parkinson's Disease. Mov Disord. 2020;35:2028-37.

33. Haroon E, Welle JR, Woolwine BJ, Goldsmith DR, Baer W, Patel T, et al. Associations among peripheral and central kynurenine pathway metabolites and inflammation in depression. Neuropsychopharmacology. 2020;45:998-1007.

34. Felger JC, Haroon E, Patel TA, Goldsmith DR, Wommack EC, Woolwine BJ, et al. What does plasma CRP tell us about peripheral and central inflammation in depression? Mol Psychiatry. 2020;25:1301-11.

35. Raison CL, Dantzer R, Kelley KW, Lawson MA, Woolwine BJ, Vogt G, et al. CSF concentrations of brain tryptophan and kynurenines during immune stimulation with IFN-alpha: relationship to CNS immune responses and depression. Mol Psychiatry. 2010;15:393-403.

36. Pu J, Liu Y, Zhang H, Tian L, Gui S, Yu Y, et al. An integrated meta-analysis of peripheral blood metabolites and biological functions in major depressive disorder. Mol Psychiatry. 2020;10:1-12.

37. Remus JL, Dantzer R. Inflammation models of depression in rodents: relevance to psychotropic drug discovery. Int J Neuropsychopharmacol. 2016;19:9.

38. Tawfik DM, Lankelma JM, Vachot L, Cerrato E, Pachot A, Wiersinga WJ, et al. Comparison of host immune responses to LPS in human using an immune profiling panel, in vivo endotoxemia versus ex vivo stimulation. Sci Rep. 2020;10:9918.

39. Walker AK, Budac DP, Bisulco S, Lee AW, Smith RA, Beenders B, et al. NMDA receptor blockade by ketamine abrogates lipopolysaccharide-induced depressivelike behavior in C57BL/6J mice. Neuropsychopharmacology. 2013;38:1609-16. 
40. Caparros-Gonzalez RA, Romero-Gonzalez B, Strivens-Vilchez H, Gonzalez-Perez R, Martinez-Augustin O, Peralta-Ramirez MI. Hair cortisol levels, psychological stress and psychopathological symptoms as predictors of postpartum depression. PLoS ONE. 2017;12:e0182817.

41. Furman D, Campisi J, Verdin E, Carrera-Bastos P, Targ S, Franceschi C, et al. Chronic inflammation in the etiology of disease across the life span. Nat Med. 2019;25:1822-32.

42. The American College of Obstetricians and Gynecologists Committee. The American College of Obstetricians and Gynecologists Committee Opinion no. 630. Screening for perinatal depression. Obstet Gynecol. 2015;125:1268-71.

43. Goodman JH. Women's attitudes, preferences, and perceived barriers to treatment for perinatal depression. Birth. 2009;36:60-69.

44. DellaGioia N, Hannestad J. A critical review of human endotoxin administration as an experimental paradigm of depression. Neurosci Biobehav Rev. 2010;34: 130-43.

45. Krishnan V, Nestler EJ. Animal models of depression: molecular perspectives. Curr Top Behav Neurosci. 2011;7:121-47.

\section{ACKNOWLEDGEMENTS}

The study was funded by the National Institutes of Mental Health (NIMH R01 MH104622). The clinical trial identifier is NCT02566980. We wish to thank the pregnant women and their families who participated in the study and all the medical staff attending the maternal health and Obstetrics and Gynecology clinics at Spectrum Health and the clinicians from Pine Rest Christian Mental Health Services who administered the SCID interview for all the participants in Grand Rapids, Michigan, USA.

\section{AUTHOR CONTRIBUTIONS}

QS data analysis and interpretation of data, drafting the article. ZM statistical consultation, editing the article. SK sample analysis. MLEG study concept and design, critical revision of the manuscript. LS clinical conduct, recruitment and assessment of patients, critical revision of the manuscript. SK sample analysis. ATF overseeing clinical conduct, critical revision of the manuscript. RL overseeing clinical conduct, critical revision of the manuscript. TTP study concept and design, critical revision of the manuscript. EA study concept and design, overseeing clinical conduct, critical revision of the manuscript, drafting the initial article. LB study concept and design, data analysis, review of statistical analysis, drafting the initial manuscript, funding acquisition.

\section{COMPETING INTERESTS}

Dr. Achtyes has served on advisory boards for Alkermes, CAPNOS Zero (unpaid), Indivior, Janssen, Lundbeck/Otsuka, Neurocrine Biosciences, Roche, Sunovion and Teva and reports previous stock holdings in AstraZeneca, Johnson \& Johnson, Moderna, and Pfizer. Dr. Achtyes has received research support from Alkermes, Astellas, Biogen, Boehringer-Ingelheim, InnateVR, Janssen, National Network of Depression Centers, Neurocrine Biosciences, Novartis, Otsuka, Pear Therapeutics, Takeda, and the Vanguard Research Group. All the other authors declare that they have no known competing financial interests or personal relationships that could have appeared to influence the work reported in this paper.

\section{ADDITIONAL INFORMATION}

Supplementary information The online version contains supplementary material available at https://doi.org/10.1038/s41398-022-01801-8.

Correspondence and requests for materials should be addressed to Lena Brundin.

Reprints and permission information is available at http://www.nature.com/ reprints

Publisher's note Springer Nature remains neutral with regard to jurisdictional claims in published maps and institutional affiliations.

Open Access This article is licensed under a Creative Commons Attribution 4.0 International License, which permits use, sharing, adaptation, distribution and reproduction in any medium or format, as long as you give appropriate credit to the original author(s) and the source, provide a link to the Creative Commons license, and indicate if changes were made. The images or other third party material in this article are included in the article's Creative Commons license, unless indicated otherwise in a credit line to the material. If material is not included in the article's Creative Commons license and your intended use is not permitted by statutory regulation or exceeds the permitted use, you will need to obtain permission directly from the copyright holder. To view a copy of this license, visit http://creativecommons. org/licenses/by/4.0/.

(c) The Author(s) 2022 